\title{
Levantamento de Parasitos Gastrintestinais Diagnosticados em Ovinos pelo Laboratório de Doenças Parasitárias da Universidade Federal de Pelotas (Brasil), nos Anos de 2015 a 2017
}

\author{
Alexsander Ferraz ${ }^{1 *}$, Tanize Angonesi de Castro $^{2}$, Tainá Ança Evaristo ${ }^{3}$, Ana Lúcia Coelho \\ Recuero $^{2}$, Paola Renata Joanol Dallmann ${ }^{3}$, Jaqueline Freitas Motta ${ }^{1} \&$ Leandro Quintana Nizoli $^{4}$ \\ ${ }^{1}$ Doutorando(a) do Programa de Pós-graduação em Veterinária da Universidade Federal de Pelotas. \\ ${ }^{2}$ Residente Multiprofissional área de Doenças e Zoonoses Parasitárias, Universidade Federal de Pelotas. \\ ${ }^{3}$ Acadêmica do curso de Medicina Veterinária da Universidade Federal de Pelotas. \\ ${ }^{4}$ Docente do curso de Medicina Veterinária da Universidade Federal de Pelotas, Departamento de Veterinária Preventiva.
}

*E-mail para correspondência: xanderferraz@yahoo.com.br

\begin{abstract}
RESUMO
Foram examinadas, pelo Laboratório de Doenças Parasitárias (LADOPAR) da Universidade Federal de Pelotas, 2.680 amostras de fezes de ovinos, advindas da região sul do estado do Rio Grande do Sul, Brasil, entre os anos de 2015 a 2017. A técnica coproparasitológica de Gordon \& Whitlock (1939) foi utilizada para o diagnóstico. Das amostras analisadas, 86,5\% apresentaram-se positivas para algum helminto gastrintestinal e oocistos de protozoários, sendo a infecção mais prevalente por ovos da ordem Strongylida. Através dos resultados obtidos, conclui-se que helmintoses gastrintestinais estão presentes nas criações de ovinos da região sul do estado e, através de exames coprológicos, é possível implementar medidas sanitárias e preventivas nos rebanhos.
\end{abstract}

Palavras-chave: Fezes, Helmintos, Ruminantes.

\section{ABSTRACT}

Survey of gastrointestinal parasites diagnosed in sheep by the Laboratory of Parasite Diseases of the Federal University of Pelotas (Brazil), from 2015 to 2017. A total of 2,680 samples of sheep feces from the southern region of the State of Rio Grande do Sul, Brazil, from 2015 to 2017 were examined by the Laboratory of Parasitic Diseases (LADOPAR) of the Federal University of Pelotas. Gordon \& Whitlock's (1939) coproparasitological technique was used for the diagnosis. 86.5\% of the samples analyzed were positive for some gastrointestinal helminth and protozoan oocysts, being the most prevalent infection by eggs of Strongylida order. Through the results obtained, it is concluded that gastrointestinal helminths are present in the sheep farms of the southern region of the state and, through coprological exams, it is possible to implement sanitary and preventive measures in the herds.

Keywords: Feces, Helminths, Ruminants. 
O Brasil possui elevado potencial para tornar-se importante produtor mundial de ovinos, com extensão territorial e clima tropical favoráveis a esta espécie animal. No entanto, as parasitoses gastrintestinais ainda determinam um fator de entrave para o desenvolvimento de sua total potencialidade e expansão neste ramo (Siqueira, 1999; Vieira, 2003).

O estado do Rio Grande do Sul detém o maior rebanho ovino do país, totalizando um efetivo de 3.496.904 animais, onde esta atividade possui destaque por sua importância, tanto econômica quanto cultural (IBGE, 2016). No entanto, as parasitoses por helmintos gastrintestinais representam o maior e mais grave problema sanitário em pequenos ruminantes (Vieira, 2003; Amarante, 2014), determinando importantes perdas econômicas na produção de ovinos devido a retardos no crescimento, perda de peso, queda da produção de leite, redução da qualidade da carne e lã, elevada taxa de morbidade e, em casos mais graves, mortalidade dos animais parasitados (Guimarães et al., 2011; Vilela et al., 2012).

As infecções parasitárias gastrintestinais em ovinos são, geralmente, de natureza mista, ou seja, várias espécies de nematódeos podem parasitar simultaneamente os animais e, para essa diversidade de espécies, alguns fatores podem ser considerados, como frequência de tratamentos com anti-helmíntico, tipo de manejo e as condições ambientais (Cenci et al., 2007; Amarante, 2014). Ainda de acordo com Amarante et al. (2004), para se obter maior êxito no combate aos helmintos, é necessário realizar um controle embasado no conhecimento dos tipos de nematódeos que acometem os animais da região, bem como sua epidemiologia. Desse modo, um adequado sistema de manejo zootécnico e sanitário, associado aos estudos epidemiológicos destes parasitos, pode diminuir a utilização de medicamentos nos animais, minimizando a possibilidade de resistência parasitária.

O presente trabalho foi realizado pelo Laboratório de Doenças Parasitárias (LADOPAR) da Universidade Federal de Pelotas com o objetivo de levantar os helmintos presentes em ovinos da região sul do Estado do Rio Grande do Sul, Brasil, entre os anos de 2015 a 2017.

Foram examinadas 2.680 amostras de fezes de ovinos recebidas no Laboratório de Doenças Parasitárias (LADOPAR) da Universidade Federal de Pelotas, Brasil, entre os anos de 2015 a 2017. As amostras eram provenientes de ovinos com diferentes idades, raças e sexo e chegavam ao laboratório armazenadas em sacos plásticos identificados, acondicionadas em caixas térmicas contendo gelo retornável, advindas de municípios da região sul do estado do Rio Grande do Sul.

A técnica coproparasitológica utilizada para diagnóstico de nematoides e cestoides gastrintestinais e oocistos de coccídeos foi a de Gordon \& Whitlock (1939), uma técnica quantitativa e qualitativa baseada na contagem de Ovos por Grama de Fezes (O.P.G.) e Oocistos por Grama de Fezes (O.O.P.G.), pelo princípio de flutuação em solução hipersaturada. A diferenciação de gêneros deu-se 
através das características morfológicas dos ovos, utilizando microscópio óptico (objetiva de 10 x).

De um total de 2.680 amostras de fezes de ovinos examinadas, 86,5\% (2.318 / 2.680) apresentavam-se positivas para algum parasito gastrintestinal, seja em infecções únicas ou associadas (Tabela 1). Destas amostras, 87,5\% (2.030) foram positivas para ovos da ordem Strongylida, 13,7\% (319) para Cestoide (Moniezia spp.), 11,4\% (266) para Strongyloides spp., 4,6\% (108) para Trichuris spp., 1,4\% (33) para Nematodirus spp., e 57,0\% (1.319) para oocistos de protozoários (Tabela 2). Ao analisarmos os anos em questão, é possível observar que a maior ocorrência se deu em 2016, com 725 amostras positivas, representando $92,6 \%$ naquele ano (Tabela 3 ).

Tabela 1. Número e percentual de amostras fecais de ovinos, positivas para diferentes helmintos gastrintestinais, diagnosticadas pelo Laboratório de Doenças Parasitárias (LADOPAR) da Universidade Federal de Pelotas entre os anos de 2015 a 2017.

\begin{tabular}{lrr}
\hline Parasitos & No Amostras positivas & \% Amostras positivas \\
\hline Ordem Strongylida & 2030 & 87,5 \\
Moniezia spp. & 319 & 13,7 \\
Strongyloides spp. & 266 & 11,4 \\
Trichuris spp. & 108 & 4,6 \\
Nematodirus spp. & 33 & 1,4 \\
Oocistos & 1319 & 57,0 \\
\hline
\end{tabular}

Ovos da ordem Strongylida foram os mais prevalentes dentre as amostras examinadas $(87,5 \%)$, valor mais elevado que o encontrado por Ahid et al. (2008), no Rio Grande do Norte, que foi 75,2\%. Os gêneros pertencentes a este grupo incluem: Cooperia, Ostertagia, Haemonchus, Trichostrongylus, Oesophagostomun (Fortes, 1997). Contudo, a diferenciação destes gêneros não foi possível devido à maioria dos proprietários não requisitarem a realização de coprocultura.

Tabela 2. Amostras positivas no diagnóstico coproparasitológico de Gordon \& Withlock (1939) para helmintos gastrintestinais por ano pesquisado. Laboratório de Doenças Parasitárias (LADOPAR) da Universidade Federal de Pelotas (2015 - 2017).

\begin{tabular}{lcccc}
\hline Parasitos & $\mathbf{2 0 1 5}$ & $\mathbf{2 0 1 6}$ & $\mathbf{2 0 1 7}$ & Positivos \\
\hline Ordem Strongylida & 848 & 625 & 557 & 2030 \\
Moniezia spp. & 111 & 140 & 68 & 319 \\
Strongyloides spp. & 41 & 157 & 68 & 266 \\
Trichuris spp. & 31 & 53 & 24 & 108 \\
Nematodirus spp. & 4 & 22 & 7 & 33 \\
Oocistos & 498 & 435 & 386 & 1319 \\
\hline
\end{tabular}

Estes helmintos têm grande importância na ovinocultura, representando consideráveis perdas neste ramo, além de serem muito comuns nesta espécie animal, como demonstrado em estudos conduzidos por Rissi et al. (2010) e Costa et al. (2011), que verificaram uma alta prevalência e intensidade 
de infecção por Haemonchus contortus, Trichostrongylus colubriformis e Oesophagostomum colubianum, sendo considerados os nematódeos de maior importância econômica para a exploração de ovinos na região central do Rio Grande do Sul e Nordeste, respectivamente, podendo-se afirmar que Haemonchus contortus é a principal espécie que parasita ovinos no país (Echevarria et al., 1996; Ramos et al., 2004; Rocha et al., 2004).

Tabela 3. Número e percentual de amostras positivas e negativas no diagnóstico coproparasitológico de Gordon \& Withlock (1939) para helmintos gastrintestinais por ano pesquisado. Laboratório de Doenças Parasitárias (LADOPAR) da Universidade Federal de Pelotas (2015 - 2017).

\begin{tabular}{lccc}
\hline Ano & Positivas (\%) & Negativas (\%) & Total \\
\hline 2015 & $963(83,6 \%)$ & $189(16,4 \%)$ & 1152 \\
2016 & $725(92,6 \%)$ & $58(7,4 \%)$ & 783 \\
2017 & $630(84,5 \%)$ & $58(7,4 \%)$ & 745 \\
\hline
\end{tabular}

Todas as 319 amostras positivas para cestódeos apresentaram ovos do gênero Moniezia spp., comumente encontrada no intestino delgado de ovinos, sendo $M$. expansa descrita como a espécie preferencial nestes animais (Amarante, 2014); resultado este superior ao descrito por Ahid et al. (2008) e Brito et al. (2009) na região nordeste do país.

Já os ovos de Strongyloides spp. apresentaram uma frequência de 11,4\% dentre os três anos estudados, valor inferior ao encontrado por Maciel et al. (2014) ao estudar a fauna helmintológica de ovinos em Jaboticabal, estado de São Paulo.

Do total de amostras, 4,6\% (108/2.680) continham ovos de Trichuris spp., que se apresentam bioperculados e larvados em sua forma infectante. No Brasil, três espécies de Trichuris foram registradas em ovinos: T. ovis, T. globulosa e T. discolor, posto que as infecções por Trichuris spp. são usualmente leves e não chegam a causar alterações patológicas perceptíveis (Amarante, 2014).

De acordo com Ramos et al. (2004) e Mattos \& Hoffman (2010), é muito comum a ocorrência de Nematodirus spp. no sul do Brasil, porém encontramos apenas 1,4\% de ovos deste parasito entre as amostras analisadas.

O parasitismo concorrente de nematoides e coccídeos em ovinos é muito frequente, o que, geralmente, exacerba seus efeitos (Hasslinger et al., 1993), propiciando a contaminação do ambiente e, consequentemente, a reinfecção dos animais (Martins et al., 2011). Neste estudo, mais da metade das amostras coprológicas foram positivas para oocistos de coccídeos $(57,0 \%)$, corroborando com os dados obtidos por Brito et al. (2009), que encontrou 58,8\% de amostras contaminadas. Estes oocistos protozoários pertencem, possivelmente, ao gênero Eimeria spp., muito comum em ruminantes. São descritas 15 espécies do gênero Eimeria spp. que parasitam ovinos, sendo a espécie mais comum a $E$. 
ovinoidalis, considerada como uma das mais patogênicas e associada ao aparecimento da doença em borregos (Platzer et al., 2005; Amarante, 2014; Brinker et al., 2014), porém, a coccidiose geralmente ocorre como o resultado de uma infecção mista e pode-se considerar extremamente raro o aparecimento de uma infecção causada por uma única espécie (Abdulkerim, 2009).

Além disso, as verminoses são assinaladas por serem de difícil controle, sejam pelas poucas informações sobre o correto período dos tratamentos ou a escolha das drogas antiparasitárias (Climeni, 2008). A frequente utilização de vermífugos pela grande maioria dos produtores, pelos menos $74 \%$ deles utilizam vermífugos mais de três vezes ao ano no RS, é atribuída às incertezas do tratamento e às perdas ocasionadas.

A maior contagem do número de ovos deve ser utilizada como apoio ao controle estratégico para redução da carga parasitária, especialmente na seca, quando as fases imaturas estarão susceptíveis à destruição por dessecação no ambiente, e como recurso que precede e acompanha as dosificações antihelmínticas nos rebanhos da região (Ahid et al., 2008).

\section{CONCLUSÃO}

Com base nos resultados encontrados no presente trabalho pode-se concluir que helmintos gastrintestinais e coccídeos acometem ovinos criados na região sul do Rio Grande do Sul. Tais resultados demonstram a importância em se realizar um monitoramento das contagens de ovos por grama de fezes (OPG) dos rebanhos desta região, possibilitando tratamento adequado aos animais acometidos e a implementação de medidas sanitárias preventivas.

\section{REFERÊNCIAS BIBLIOGRÁFICAS}

Abdulkerim, D. 2009. Coccídiose ovina: revisão bibliográfica. Global Veterinary Services Manager 4: 4-11.

Ahid, S.M.M.; Suassuna, A.C.D.; Maia, M.B.; Costa, V.M.M. \& Soares, H.S. 2008. Parasitos gastrintestinais em caprinos e ovinos da região oeste do Rio Grande do Norte, Brasil. Ciência Animal Brasileira 9(1): 212218.

Amarante, A.F.T.; Bricarello, P.A.; Rocha, R.A. \& Gennari, S.M. 2004. Resistance of santa ines, Suffolk and ile de France lambs to naturally acquired gastrointestinal nematode infections. Veterinary Parasitology 
120(1-2): 91-106.

Amarante, A.F.T. 2014. Classe cestoda. In: Os Parasitas de Ovinos [online]. São Paulo, Editora UNESP, 263p.

Brinker, J.C.; Rocha, A.G.; Bisol, J. \& Araújo, F.A.P. 2014. Identificação de espécies de Eimeria spp. em ovinos participantes na 33a Exposição Internacional de Animais no município de Esteio, RS. Revista Agrocientífica 1(1): 61-68.

Brito, D.R.B.; Santos, A.C.G.; Teixeira, W.C. \& Guerra, R.M.S.N.C. 2009. Parasitos gastrintestinais em caprinos e ovinos da microrregião do Alto Mearim e Grajaú, no estado do Maranhão, Brasil. Ciência Animal Brasileira 10(3): 967-974.

Cenci, F.B.; Louvandini, H.; Mcmanus, C.M.; Dell' Porto, A.; Costa, D.M.; Araujo, S.C.; Minho, A.P. \& Abdalla, A.L. 2007. Effects of condensed tannin from Acacia mearnsii on sheep infected naturally with gastrointestinal helminthes. Veterinary Parasitology 144(1-2): 132-137.

Climeni, B.S.O. 2008. Hemoncose ovina. Revista Cientifica Eletrônica de Medicina Veterinária 6(11): 1-7

Costa, V.M.M.; Simões, S.V.D. \& Riet-Correa, F. 2011. Controle das parasitoses gastrintestinais em ovinos e caprinos na região semiárida do Nordeste do Brasil. Pesquisa Veterinária Brasileira 31(1): 65-71

Echevarria, F.; Borba, M.F.S.; Pinheiro, A.C.; Waller, P.J. \& Hansen, J.W. 1996. The Prevalence of Anthelmintic Resistance in Nematode Parasites of Sheep in Southern Latin America: Brazil. Veterinary Parasitology 62(3-4): 199-206.

Fortes, E. 1997. Parasitologia Veterinária. 3ed. São Paulo, Ícone, 686p.

Gordon, H.M.C.L. \& Whitlock, H.V. 1939. A new techinique for counting nematode eggs in sheep feces. Journal Council Scientific Industry Research Australia 12(1): 50-52.

Guimarães, A.S.; Gouveia, A.M.G.; Carmo, F.B.; Gouveia, G.C.; Silva, M.X.; Vieira, L.S. \& Molento, M.B. 2011. Management practices to control gastrointestinal parasites in dairy and beef goats in Minas Gerais, Brazil. Veterinary Parasitology 176(2-3): 265-269.

Hasslinger, M.A.; Schenkel, F.; Ogaylat, S. \& Ulbricht, G. 1993. Important endoparasites in sheep and goat flocks in Jordan. Journal of Veterinary Medicine 40(5): 329-336.

IBGE. 2016. Sistema IBGE de Recuperação Automática. Banco de Dados Agregados. Disponível em: 
<http://www.sidra.ibge.gov.br/bda/tabela/listabl.asp?c=3939\&z=t\&o=24>. Acesso em: 16 abr. 2018.

IBGE. 2016. Instituto Brasileiro de Geografia e Estatística. Rio Grande do Sul. Disponível em: <https://cidades.ibge.gov.br/brasil/rs/pesquisa/18/16442>. Acesso em: 10 abr. 2018.

Maciel, W.G.; Felippelli, G.; Lopes, W.D.Z.; Teixeira, W.F.P.; Cruz, B.C.; Santos, T.R.; Buzzulini, C.; Favero, F.; Gomes, L.C.; Oliveira, G.P.; Costa, A.J. \& Matos, L.V.S. 2014. Fauna helmintológica de ovinos provenientes da microrregião de Jaboticabal, estado de São Paulo, Brasil. Ciência Rural 44(3): 492-497.

Martins, G.F.; Moura, M.S.; Cabral, D.D. \& Souza, R.R. 2011. Frequência de oocisto de Eimeria spp. em ovinos de propriedades rurais do Município de Uberlândia-MG. Pubvet 5(7): 1034-1041.

Mattos, M.J.T. \& Hoffmann, R.P. 2010. Diagnóstico Laboratorial em Helmintoses. 3 ed. Porto Alegre, Gráfica Ufrgs. 63p.

Platzer, B.; Prosl, H.; Cieslicki, M. \& Joachim, A. 2005. Epidemiology of Eimeria infections in an Austrian milking sheep flock and control with diclazuril. Veterinary Parasitology 129(1-2): 1-9.

Ramos, C.I.; Bellato, V.; Souza, A.P.; Avila, V.S.; Coutinho, G.C. \& Dalagnol, C.A. 2004. Epidemiologia das helmintoses gastrintestinais de ovinos no Planalto Catarinense. Ciência Rural 34(6): 1889-95.

Rissi, D.R.; Pierezan, F.; Oliveira Filho, J.C.; Fighera, R.A.; Irigoyen, L.F.; Kommers, G.D. \& Barros, C.S.L. 2010. Doenças de ovinos da região Central do Rio Grande do Sul: 361 casos. Pesquisa Veterinária Brasileira 30(1): 21-28.

Rocha, R.A.; Amarante, A.F.T. \& Bricarello, P.A. 2004. Comparison of the susceptibility of Santa Ines and lle de France ewes to nematode parasitism around parturition and during lactation. Small Ruminant Research 55(1-3): 65-75.

Siqueira, E.R. 1999. Revista Tecnologia e Treinamento Agropecuário. Ano 3, n.10, 32p.

Vieira, L.S. 2003. Alternativas de controle da verminose gastrintestinal dos pequenos ruminantes. Sobral: Embrapa Caprinos, Circular Técnico Online, n.29, 10p.

Vilela, V.L.R.; Feitosa, T.F.; Linhares, E.F.; Athayde, A.C.; Molento, M.B. \& Azevedo, S.S. 2012. FAMACHA method as an auxiliary strategy control of gastrointestinal helminthiasis of dairy goats under semiarid conditions of Brazil northeastern. Veterinary Parasitology 190(1-2): 281-284. 